\title{
Proinflammatory and Anabolic Gene Expression Effects of Platelet-Rich Gel Supernatants on Equine Synovial Membrane Explants Challenged with Lipopolysaccharide
}

\author{
Jorge U. Carmona, ${ }^{1}$ Diana L. Ríos, ${ }^{1}$ Catalina López, ${ }^{1}$ María E. Álvarez, ${ }^{1,2}$ and Jorge E. Pérez ${ }^{2}$ \\ ${ }^{1}$ Grupo de Investigación Terapia Regenerativa, Departamento de Salud Animal, Universidad de Caldas, Manizales, Colombia \\ ${ }^{2}$ Grupo de Investigación Biosalud, Departamento de Ciencias Básicas para la Salud, Universidad de Caldas, Manizales, Colombia \\ Correspondence should be addressed to Jorge U. Carmona; carmona@ucaldas.edu.co
}

Received 4 March 2017; Revised 10 May 2017; Accepted 5 June 2017; Published 6 July 2017

Academic Editor: Lorraine M. Sordillo

Copyright (C) 2017 Jorge U. Carmona et al. This is an open access article distributed under the Creative Commons Attribution License, which permits unrestricted use, distribution, and reproduction in any medium, provided the original work is properly cited.

\begin{abstract}
Platelet-rich plasma (PRP) preparations are used in horses with osteoarthritis (OA). However, some controversies remain regarding the ideal concentration of platelets and leukocytes to produce an adequate anti-inflammatory and anabolic response in the synovial membrane. The aims of this study were to study the influence of leukoconcentrated platelet-rich gel (Lc-PRG) and leukoreduced platelet-rich gel (Lr-PRG) supernatants on the quantitative expression of some proinflammatory and anabolic genes in equine synovial membrane explants (SMEs) challenged with lipopolysaccharide (LPS). SMEs from six horses were cultured over $96 \mathrm{~h}$. Then, SMEs were harvested for RNA extraction and quantitative gene expression analysis by RT-qPCR for nuclear factor kappa B $(\mathrm{NF} \kappa \mathrm{B})$, matrix metalloproteinase 13 (MMP-13), a disintegrin and metalloproteinase with thrombospondin motifs 4 (ADAMTS-4), collagen type I alpha 1 (COL1A1), collagen type II alpha 1 (COL2A1), and cartilage oligomeric matrix protein (COMP). The 25\% and 50\% Lc-PRG supernatants led to downregulation of NFאB, MMP-13, ADAMTS-4, COL1A1, COL2A1, and COMP in SMEs. LrPRG supernatants (particularly at the 50\% concentration) induced downregulation of NF $\kappa$ B, MMP-13, ADAMTS-4, and COL1A1 and upregulation of COL2A1 and COMP. Lr-PRG supernatants should be used for the treatment of inflammatory arthropathies in horses because they have anti-inflammatory and anabolic effects in the synovial membrane.
\end{abstract}

\section{Introduction}

Currently, platelet-rich plasma (PRP) and other plateletrelated products have emerged as a therapeutic option for the treatment of osteoarthritis (OA) in humans $[1,2]$ and horses [2-6]. However, there are several PRP preparations with different cellular (platelets and white blood cells (WBCs)) and molecular (growth factors (GFs) and cytokines) profiles, which undoubtedly produce different joint tissue responses after they come into contact with these substances $[1,2,7$, 8].

Although not perfected, there is a standard classification for liquid PRP preparations, which comprises two categories: (1) leukoreduced PRP (Lr-PRP) and (2) leukoconcentrated PRP (Lc-PRP) preparations [9]. In a general fashion, LrPRP preparations are represented by cell concentrates with variable counts of platelets and without or with negligible numbers of WBCs, whereas Lc-PRP preparations are hemoderivatives with high concentration of leukocytes [9]. Notably, once any PRP preparation is mixed with an activating substance, like calcium salts or thrombin, it is polymerized into a platelet-rich gel (PRG), which is a live biological scaffold with the capacity to retain and release GFs over time [10].

There are controversies about which PRP preparation (either Lr-PRP or Lc-PRP) is better to treat osteoarthritic patients $[4,11,12]$. Some investigators favor the use of Lr-PRP [13], whereas others recommend using Lc-PRP preparations [14]. However, additional research is necessary to address this concern. In line with this, we have performed an in vitro study to evaluate the effects of different concentrations (25 and 50\%) of Lr-LPG and Lc-PRG supernatants over a $96 \mathrm{~h}$ period on anabolic and proinflammatory gene expression 


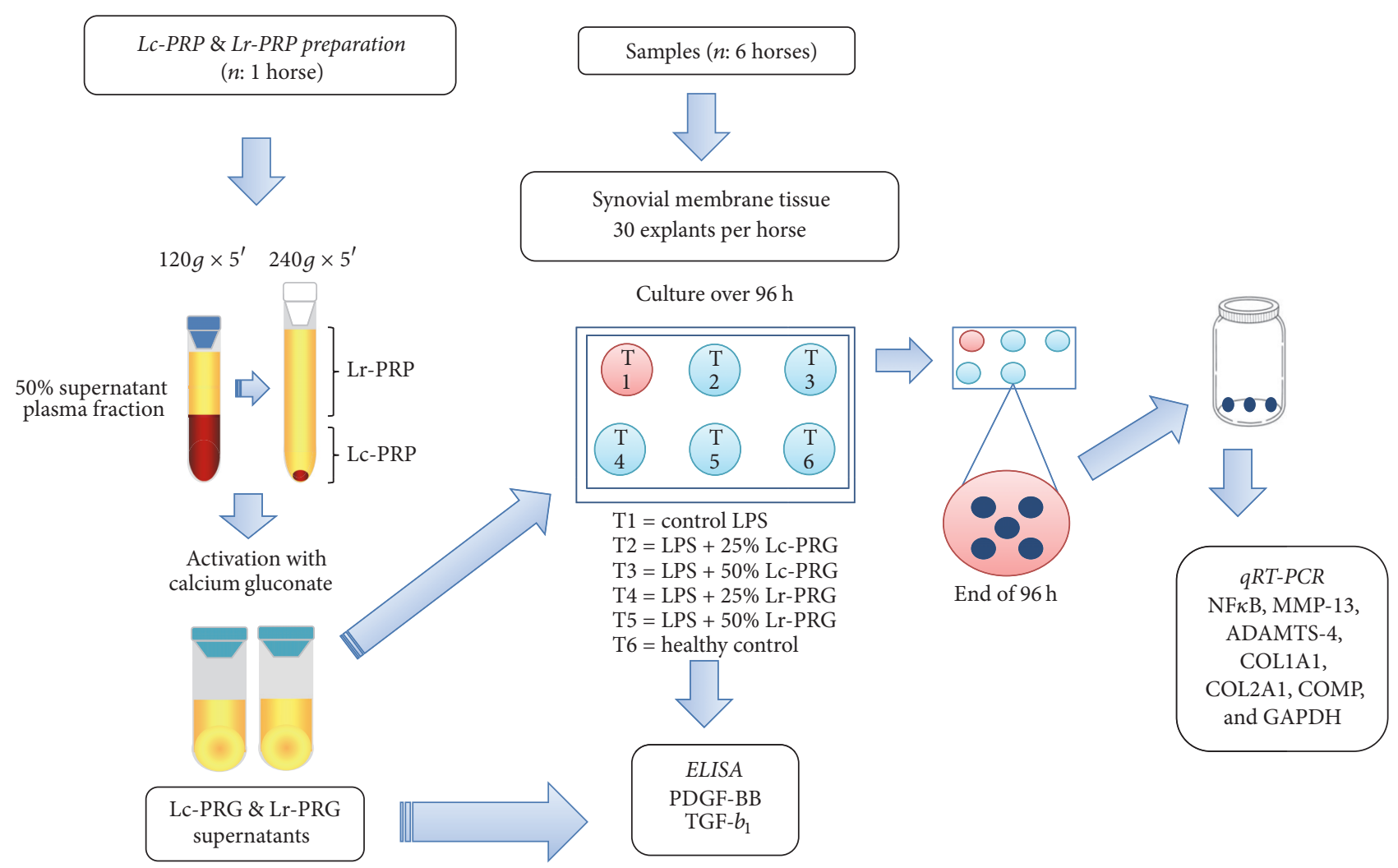

FIGURE 1: Schematic workflow of the experiments in the study.

in synovial membrane explants (SMEs) challenged with lipopolysaccharide (LPS).

We evaluated the expression of some key genes (nuclear factor kappa $\mathrm{B}(\mathrm{NF} \kappa \mathrm{B})$, matrix metalloproteinase 13 (MMP-13), a disintegrin and metalloproteinase with thrombospondin motifs 4 (ADAMTS-4), collagen type I alpha 1 (COL1A1), collagen type II alpha 1 (COL2A1), and cartilage oligomeric matrix protein (COMP)) implicated in joint homeostasis and pathology. Our hypotheses were that both PRG supernatants at different concentrations produce diverse gene expression changes in inflamed SMEs.

\section{Materials and Methods}

This study was approved by the committee on animal experimentation at the authors' institution.

2.1. Samples. Synovial membrane samples from the metacarpophalangeal joints from six horses, aged 5 to 11 years, were included in this study. The samples were taken from horses that were free from musculoskeletal disease and euthanized by a pentobarbital intravenous overdose for other medical reasons. All metacarpophalangeal joints were radiographed and macroscopically evaluated to exclude horses with OAassociated joint changes.

2.2. Lr-PRP and Lc-PRP Preparation. Venous blood from one clinically healthy mare was used in order to avoid variability in the GF and cytokine concentrations in the PRG supernatants used in the experiments. Platelet concentrates were obtained through a manual double centrifugation tube method [15]. Blood was drawn from jugular venipuncture and deposited in $4.5 \mathrm{~mL}$ tubes with sodium citrate solution (BD Vacutainer ${ }^{\circledR}$, Becton Drive, Franklin Lakes, NJ, USA). After centrifugation at $120 \times \mathrm{g}$ for 5 minutes, the first $50 \%$ of the top supernatant plasma fraction, adjacent to the buffy coat, was collected. This fraction was centrifuged at $240 \times \mathrm{g}$ for 5 minutes, and then the bottom quarter of the fraction was collected [15]. This fraction was designated as Lc-PRP. The upper plasma fraction was designated as Lr-PRP (Figure 1). Whole blood and both PRP preparations were analyzed for platelet and WBC counts using an impedance-based hematology device (Celltac- $\alpha$ MEK 6450, Nihon Kohden, Japan).

Both PRP preparations were activated with calcium gluconate (Ropsohn Therapeutics, Ltda., Bogotá, Colombia) (ratio $1: 10$ ) and incubated at $37^{\circ} \mathrm{C}$ for $1 \mathrm{~h}$ until clot retraction occurred. Fresh Lr-PRG and Lc-PRG supernatants were used to add to the culture media at two concentrations (25\% and 50\%). Aliquots of both PRG supernatants were frozen at $-86^{\circ} \mathrm{C}$ for later quantification of platelet-derived growth factor $\mathrm{BB}$ (PDGF-BB) and transforming growth factor beta 1 (TGF- $\left.\beta_{1}\right)$.

2.3. Synovial Membrane Explants Culture and LPS Challenge. Synovial membrane samples were obtained aseptically, and circular $4 \mathrm{~mm}$ diameter explants were obtained using a disposable biopsy punch (KAI Medical, Solingen, Germany). 
TABLE 1: Genes and primer sequences used in the study.

Targeted genes

GAPDH, glyceraldehyde-3-phosphate dehydrogenase 6

$\mathrm{NF} \kappa \mathrm{B}$, nuclear factor of kappa light polypeptide gene enhancer in B-cells-like 1

MMP 13, matrix metallopeptidase 13 (collagenase 3) 7

ADAMTS 4, metallopeptidase with thrombospondin type 1 motif 4

COL1A1, collagen, type I, alpha 111

COL2A1, collagen, type II, alpha 16

COMP, cartilage oligomeric matrix protein 21
Primer sequences $\left(5^{\prime} \rightarrow 3^{\prime}\right)$

Forward TCCCTGCTTCTACTGGTGCT

Reverse TGACAAAGTGGTCGTTGAGG

Forward CGATTTCGATATGGCTGTGA

Reverse CACCTTCTTCAGCTCCTTGG

Forward GCATTCAAAAAGGCCTTCAA

Reverse GGAAGCACAAAGTGGCTTTT

Forward TGTCAGCTTGGTGGTGACTC

Reverse GTTGAAGACATGGCCCAGTT

Forward AGCCAGCAGATCGAGAACAT

Reverse CTGGCCACCATACTCGAACT

Forward ACGTCCAGATGACCTTCCTG

Reverse GTCCACACCAAATTCCTGCT

Forward CCACGTGAATACGGTCACAG

Reverse TAGGAACCAGCGGTAGGATG
SMEs were cultured in culture media standard as described in [12]. After $24 \mathrm{~h}, \mathrm{SMEs}$ were challenged with $100 \mathrm{ng} / \mathrm{mL}$ of LPS (Sigma-Aldrich, St Louis, MO, USA) to induce inflammatory/catabolic damage to these tissues [16].

2.4. Study Design. A total of 30 SMEs were obtained from each horse. The study design included the evaluation of six experimental groups using five SMEs per group from each horse as follows: one SME healthy control group without LPS and without the addition of any PRG supernatant, one SME control group challenged with LPS and without the addition of any PRG supernatant, and four SME groups cultured with Lc-PRG and Lr-PRG supernatants at two different concentrations (25\% and 50\%) and with LPS. After $1 \mathrm{~h}$ of incubation, Lc-PRG and Lr-PRG supernatants were added in order to obtain concentrations at $25 \%$ and $50 \%$. All SME groups were cultured at $96 \mathrm{~h}$, after which they were deposited in an RNA conserving solution (RNAlater, Life Technologies, Carlsbad, CA, USA) for quantitative gene expression of NF $\kappa$ B, MMP-13, ADAMTS-4, COL1A1, COL2A1, and COMP. A schematic diagram (Figure 1) summarizes the study design and methodology.

2.5. ELISA Analysis. Lc-PRG and Lr-PRG supernatants were used to determine the concentration of PDGF-BB and TGF$\beta_{1}$ by ELISA in duplicate. All proteins were assayed using commercial ELISA kits from R\&D Systems (Minneapolis, MN, USA). PDGF-BB (Human PDGF-BB DuoSet, DY220) and TGF- $\beta_{1}$ (Human TGF- $\beta_{1}$ DuoSet, DY240E) were determined using human antibodies due to the high sequence homology between these proteins in humans and horses $[17,18]$. Furthermore, these kits have been used for the same purposes in other equine PRP studies [19, 20]. Standards provided for each ELISA kit were used to prepare each standard curve following the manufacturer's instructions. Absorbance readings were performed at $450 \mathrm{~nm}$.

2.6. Quantitative Gene Expression Evaluation. Synovial membrane samples were prepared for RNA extraction as described previously [12]. Samples were diluted to a concentration of $5 \mathrm{ng} / \mu \mathrm{L}$ of RNA. The samples were assayed for quantitative gene expression levels in a qRT-PCR device (StepOnePlus Real-Time PCR System, Life Technologies, Carlsbad, CA, USA) using the SuperScript III platinum SYBR Green One-Step qRT-PCR kit (Life Technologies, Carlsbad, CA, USA). Primers were used for NF $\kappa$ B, MMP-13, ADAMTS4, COL2A1, COMP, and glyceraldehyde 3-phosphate dehydrogenase (GAPDH) (Table 1). These sequences were published previously [12]. The relative change in gene expression was determined via the comparative $2^{-\Delta \Delta C T}$ method [21]. GAPDH was used as the internal control (housekeeping gene), and synovial membrane samples from all horses that were not incubated with any treatment were used as reference samples.

\section{Statistical and Data Analysis}

The statistical analysis was performed with SPSS 19.0 software (SPSS, IBM, NY, USA). The Shapiro-Wilk test was used to assess the fit of the data set to a normal distribution (goodness of fit). Data from hemoderivatives presented a normal distribution. However, data from SMEs presented a nonparametric distribution $(p<0.05)$. Several arithmetic transformations were performed to obtain normally distributed gene expression data from SMEs, but they were not successful to obtain this objective.

Platelet and WBC counts in whole blood and both PRP preparations were evaluated through a one-way analysis of variance (ANOVA), followed by Tukey's test. PDGF-BB and TGF- $\beta_{1}$ from both PRG supernatants were compared using an unpaired $t$-test. Relative gene expression data were evaluated by a rank ANOVA (Kruskal-Wallis test), followed when necessary by the Mann-Whitney $U$ test. A correlation analysis was performed to determine the Spearman correlation coefficient $(\rho)$ between the relative expression of the genes in the study. A $p$ value $<0.05$ was accepted as statistically significant for all tests. Data are presented as either mean \pm standard deviation (SD) or median (interquartile range (IR)). 


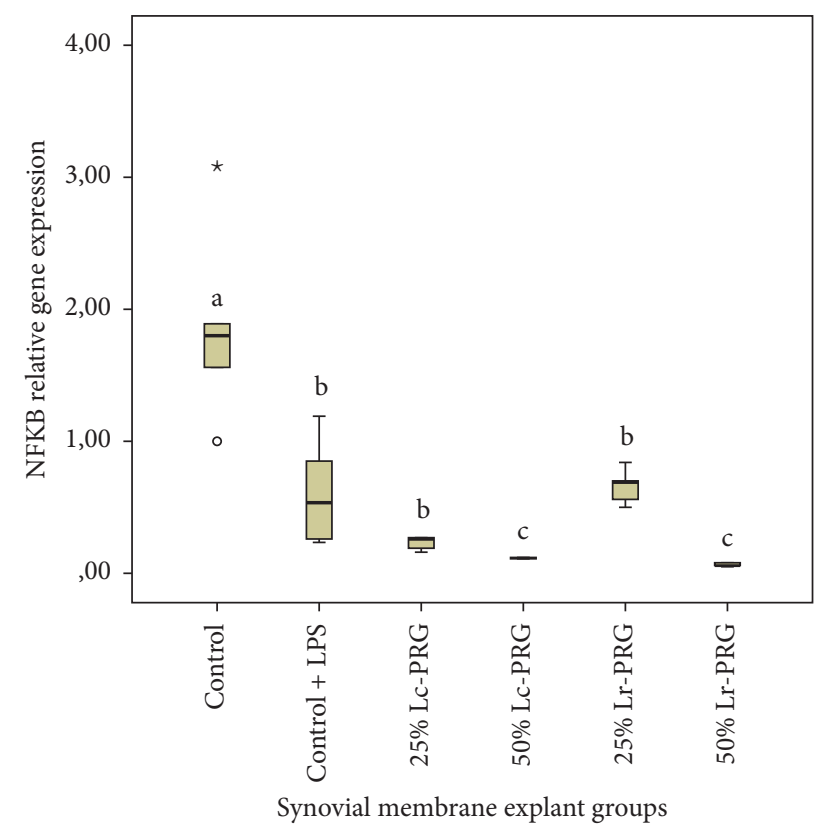

FIGURE 2: Box plot of median relative nuclear factor kappa B (NFKB) gene expression. ${ }^{\mathrm{a}-\mathrm{c}}$ Lowercase letters denote significant differences $(p>0.05)$ between synovial membrane explant (SME) groups treated with leukoconcentrated platelet-rich gel (Lc-PRG) and leukoreduced platelet-rich gel (Lr-PRG) supernatants at different concentrations (25 and 50\%). Groups with the same lowercase letter are not significantly different. $\circ$ denotes an outlier value, whereas * denotes extreme value; both symbols show nonparametric data.

\section{Results}

4.1. Cell and Growth Factor Concentration in Lc-PRP/Lc-PRG and $L r-P R P / L r-P R G$. Platelet counts were significantly $(p<$ 0.05 ) different between whole blood, Lc-PRP, and Lr-PRP, with the lowest concentration for this last hemoderivative $(97,600 \pm 3,700 \mathrm{PLT} / \mu \mathrm{L})$, followed by whole blood $(124,700$ $\pm 3,800 \mathrm{PLT} / \mu \mathrm{L})$ and $\mathrm{Lc}-\mathrm{PRP}(310,900 \pm 20,500 \mathrm{PLT} / \mu \mathrm{L})$. WBC counts were also significantly different between the evaluated groups, with a higher concentration for Lc-PRP $(36,900 \pm 4,600 \mathrm{WBC} / \mu \mathrm{L})$, followed by whole blood $(8,000$ $\pm 4,700 \mathrm{WBC} / \mu \mathrm{L})$ and $\operatorname{Lr}-\mathrm{PRP}(100 \pm 30 \mathrm{WBC} / \mu \mathrm{L})$. The TGF- $\beta_{1}$ concentration was similar between Lc-PRG (1766.3 $\pm 321.3 \mathrm{pg} / \mathrm{mL})$ and Lr-PRG $(1465.9 \pm 19.8 \mathrm{pg} / \mathrm{mL})$. PDGFBB had a significantly $(p<0.05)$ higher concentration in Lc-PRG (3067.5 $\pm 946.8 \mathrm{pg} / \mathrm{mL})$ when compared to Lr-PRG $(378.6 \pm 89.7 \mathrm{pg} / \mathrm{mL})$.

4.2. Quantitative Gene Expression. $\mathrm{NF} \kappa \mathrm{B}$ gene expression was significantly $(p<0.05)$ increased in SMEs from the control group in comparison with the SMEs of the control plus LPS group and those SME groups treated with both PRG supernatants at different concentrations (Figure 2). The expression of this gene was not significantly different between the SME control plus LPS group and the SME groups treated with both PRG supernatants at the concentration of $25 \%$. Interestingly, the SME groups treated with both PRG supernatants at the concentration of $50 \%$ showed statistically

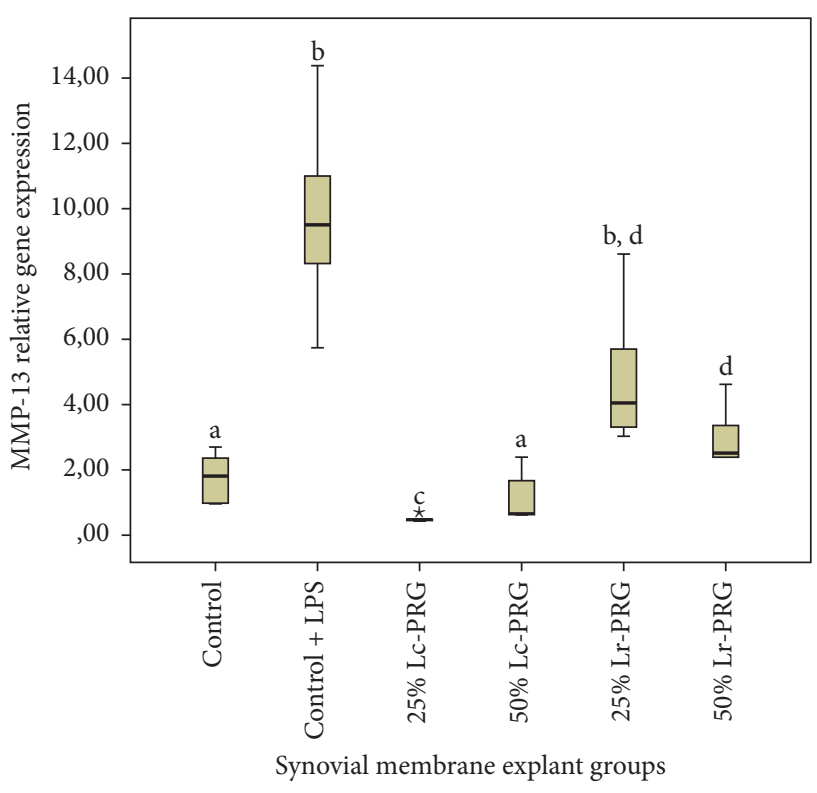

FIGURE 3: Box plot of median relative matrix metalloproteinase 13 (MMP-13) gene expression. ${ }^{a-d}$ Lowercase letters denote significant differences $(p>0.05)$ between synovial SME groups treated with Lc-PRG and Lr-PRG supernatants at different concentrations (25 and 50\%). Groups with the same lowercase letter are not significantly different. * denotes extreme value, showing nonparametric data.

significant lower $(p<0.05) \mathrm{NF} \kappa \mathrm{B}$ gene expression when compared with the other SME groups evaluated (Figure 2).

SMEs from the control group plus LPS presented significantly $(p<0.05)$ higher MMP-13 gene expression when compared to SMEs of the control group and those treated with the 25\% and 50\% Lc-PRG and 50\% Lr-PRG supernatants (Figure 3). Notably, MMP-13 gene expression was significantly lower in SMEs cultured with the $25 \%$ LcPRG supernatant when compared to the remaining SME groups (Figure 3). In general, the expression of this gene was significantly $(p<0.05)$ lower in SMEs treated with LcPRG supernatants at both concentrations when compared with SMEs treated with Lr-PRG supernatants at the same concentrations.

ADAMTS-4 gene expression was not significantly different between SMEs from the control group, the control group plus LPS, and the SMEs of the group treated with $50 \%$ LcPRG. The expression of this gene was also not significantly different between the SMEs of the control group plus LPS and those SMEs treated with both PRG supernatants at the concentration of 25\% (Figure 4). Notably, the SMEs of the groups treated with 50\% Lr-PRG supernatants showed significantly lower $(p<0.05)$ ADAMTS-4 gene expression than the remaining SME groups (Figure 4).

COL1A1 relative gene expression was significantly $(p<$ 0.05) increased in SMEs in the control group challenged with LPS in comparison with the remaining SME groups (Figure 5). Notably, the expression of this gene was significantly $(p<0.05)$ lower in all SMEs treated with both PRG supernatants at both concentrations. However, both 


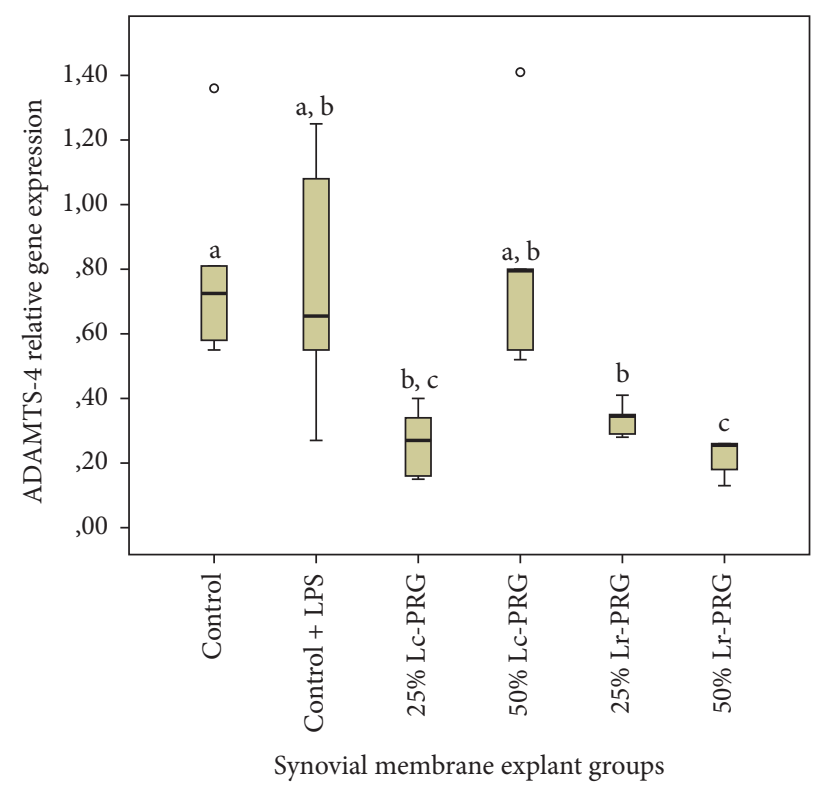

FIGURE 4: Box plot of median relative a disintegrin and metalloproteinase with thrombospondin motifs 4 (ADAMTS-4) gene expression. ${ }^{\mathrm{a}-\mathrm{c}}$ Lowercase letters denote significant differences $(p>$ 0.05) between SME groups treated with Lc-PRG and Lr-PRG supernatants at different concentrations (25 and 50\%). Groups with the same lowercase letter are not significantly different. $\circ$ denotes an outlier value, showing nonparametric data.

50\% PRG supernatants exhibited significantly lower $(p<$ $0.05)$ COL1A1 relative gene expression than the homologous substances at a concentration of $50 \%$ (Figure 5).

COL2A1 gene expression was significantly $(p<0.05)$ increased in SMEs from the control group in comparison to SMEs from the control group challenged with LPS and those SMEs cultured with both PRG supernatants at both concentrations (Figure 6). The expression of this gene was not significantly different between SMEs from the control group plus LPS and SMEs in the groups cultured with the Lr-PRG supernatants at both concentrations. Notably, SMEs cultured with Lc-PRG at both concentrations presented the most significantly reduced expression of this gene compared to the remaining SMEs groups (Figure 6).

COMP gene expression was significantly $(p<0.05)$ increased in SMEs from the control group and those cultured with 50\% Lr-PRG supernatant in comparison with the SMEs of the remaining groups (Figure 7). Interestingly, COMP gene expression in SMEs of the control group plus LPS and SMEs of the groups cultured with Lc-PRG supernatants at both concentrations and the $25 \%$ Lr-PRG supernatant were not statistically different and remained upregulated in comparison to SMEs from the control group and those cultured with the 50\% Lr-PRG supernatant (Figure 7).

A summary of the main effects affecting proinflammatory and anabolic gene expression in the SMEs is presented in Table 2.

4.3. Correlations. Significant negative correlations were observed between COLA1 and ADAMTS-4 gene expression

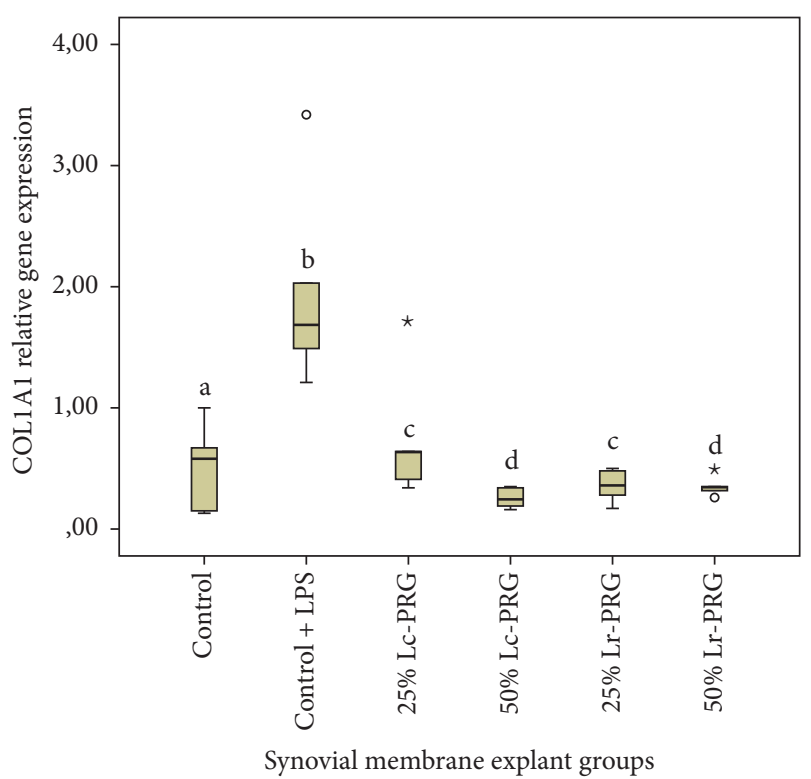

FIgURE 5: Box plot of median relative collagen type I alpha 1 (COL1A1) gene expression. ${ }^{\mathrm{a}-\mathrm{d}}$ Lowercase letters denote significant differences $(p>0.05)$ between SME groups treated with Lc-PRG and Lr-PRG supernatants at different concentrations (25 and 50\%). Groups with the same lowercase letter are not significantly different. - denotes an outlier value, whereas $\star$ denotes extreme value; both symbols show nonparametric data.

$(\rho=-0.60 ; p<0.004)$, COMP and NFאB gene expression $(\rho=-0.63 ; p<0.001)$, and COMP and ADAMTS- 4 gene expression $(\rho=-0.60 ; p<0.003)$.

\section{Discussion}

The present study aimed to determine the relative expression (either down- or upregulation) of some proinflammatory (NFאB, MMP-13, and ADAMTS-4) and anabolic (COL1A1, COL2A2, and COMP) genes implicated in OA pathophysiology in an in vitro system of equine synovitis, in which two different PRG supernatants at concentrations of $25 \%$ and $50 \%$ were evaluated. The results of this study partially indicate that inflamed synovial membrane responses to PRP preparations are different when compared to cartilage [12].

All PRG supernatants at different concentrations produced an interesting downregulation of proinflammatory genes (MMP-13 and ADAMTS-4) when compared to the gene expression observed in SMEs from the control group plus LPS. This anti-inflammatory effect was slight for the $25 \%$ Lr-PRG supernatant, moderate for the $25 \%$ Lc-PRG supernatant, and intense for the 50\% Lc-PRG and 50\% Lr-PRG supernatants. However, the Lc-PRG supernatants at both concentrations presented a slight to moderated catabolic effect, whereas the $25 \%$ Lr-PRG supernatant was slightly anabolic and the 50\% Lr-PRG was intensely anabolic (Table 2).

We observed that $\mathrm{NF} \kappa \mathrm{B}$ relative gene expression was downregulated in SMEs from the control group challenged with LPS. Interestingly, MMP-13 of the same SME group was 
TABLE 2: General proinflammatory and anabolic gene expression effects of several treatments applied to the synovial membrane explant (SME) groups in this study.*

\begin{tabular}{|c|c|c|c|c|c|c|c|}
\hline \multirow{2}{*}{ SME group } & \multicolumn{6}{|c|}{ Relative gene expression in relation to GAPDH } & \multirow{2}{*}{ Biological effect } \\
\hline & NFKB & MMP-13 & ADAMTS-4 & COL1A1 & COL2A1 & COMP & \\
\hline Control group plus LPS & $\uparrow \uparrow$ & $\uparrow \uparrow \uparrow$ & $\uparrow \uparrow$ & $\uparrow \uparrow$ & $\uparrow$ & $\uparrow$ & $\begin{array}{c}\text { Proinflammatory and catabolic } \\
\text { effect }\end{array}$ \\
\hline 25\% Lc-PRG supernatant & $\downarrow$ & $\downarrow \downarrow \downarrow$ & $\downarrow \downarrow$ & $\downarrow$ & $\downarrow \downarrow$ & $\downarrow \downarrow$ & $\begin{array}{c}\text { Moderate anti-inflammatory but } \\
\text { non anabolic effect }\end{array}$ \\
\hline $50 \%$ Lc-PRG supernatant & $\downarrow \downarrow \downarrow$ & $\downarrow \downarrow$ & $\downarrow$ & $\downarrow \downarrow$ & $\downarrow \downarrow$ & $\downarrow \downarrow$ & $\begin{array}{l}\text { Intense anti-inflammatory but } \\
\text { moderate catabolic effect }\end{array}$ \\
\hline 25\% Lr-PRG supernatant & $\downarrow$ & $\downarrow$ & $\downarrow \downarrow$ & $\downarrow$ & $\uparrow$ & $\downarrow \downarrow \downarrow$ & $\begin{array}{l}\text { Slight anti-inflammatory and } \\
\text { anabolic effect }\end{array}$ \\
\hline 50\% Lr-PRG supernatant & $\downarrow \downarrow \downarrow$ & $\downarrow$ & $\downarrow \downarrow \downarrow$ & $\downarrow \downarrow$ & $\uparrow \uparrow$ & $\uparrow \uparrow \uparrow$ & $\begin{array}{l}\text { Intense anti-inflammatory and } \\
\text { intense anabolic effect }\end{array}$ \\
\hline
\end{tabular}

${ }^{*}$ This classification was made only comparing the SME of the control group plus LPS and the SMEs groups cultured with both PRG supernatants at two concentrations plus LPS. $\uparrow=$ slight increase. $\uparrow \uparrow=$ moderate increase. $\uparrow \uparrow \uparrow=$ intense increase. $\downarrow=$ slight decrease. $\downarrow \downarrow=$ moderate decrease. $\downarrow \downarrow \downarrow=$ intense decrease.

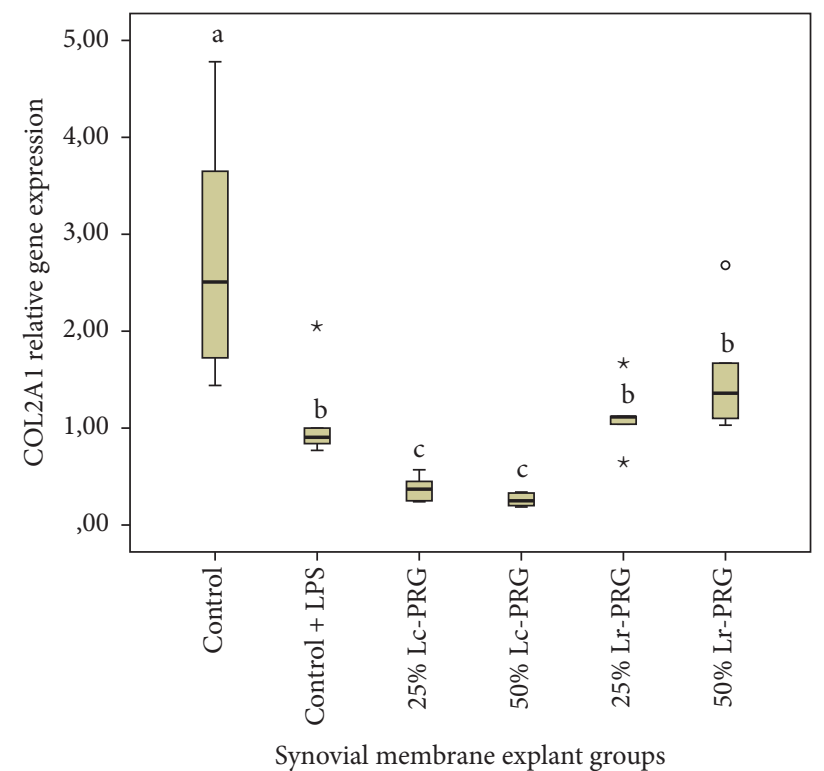

FIgURE 6: Box plot of median relative collagen type II alpha 1 (COL2A1) gene expression. ${ }^{\mathrm{a}-\mathrm{c}}$ Lowercase letters denote significant differences $(p>0.05)$ between SME groups treated with Lc-PRG and Lr-PRG supernatants at different concentrations (25 and 50\%). Groups with the same lowercase letter are not significantly different. - denotes an outlier value, whereas $\star$ denotes extreme value; both symbols show nonparametric data.

upregulated, whereas ADAMTS-4 gene expression remained slightly increased without reaching an statistically significant level. Notably, the opposite $\mathrm{NF} \kappa \mathrm{B}$ gene expression profile (upregulation) was observed in a similar in vitro study using cartilage explants [12]; however, in this last study, MMP-13 and ADAMTS-4 were also upregulated in cartilage explants challenged with LPS [12]. This finding could indicate that there is a differential response of $\mathrm{NF} \kappa \mathrm{B}$ gene expression in equine cartilage and the synovial membrane when challenged with LPS and, MMP-13, and ADAMTS-4 upregulation in SMEs challenged with LPS could be related to an alternative

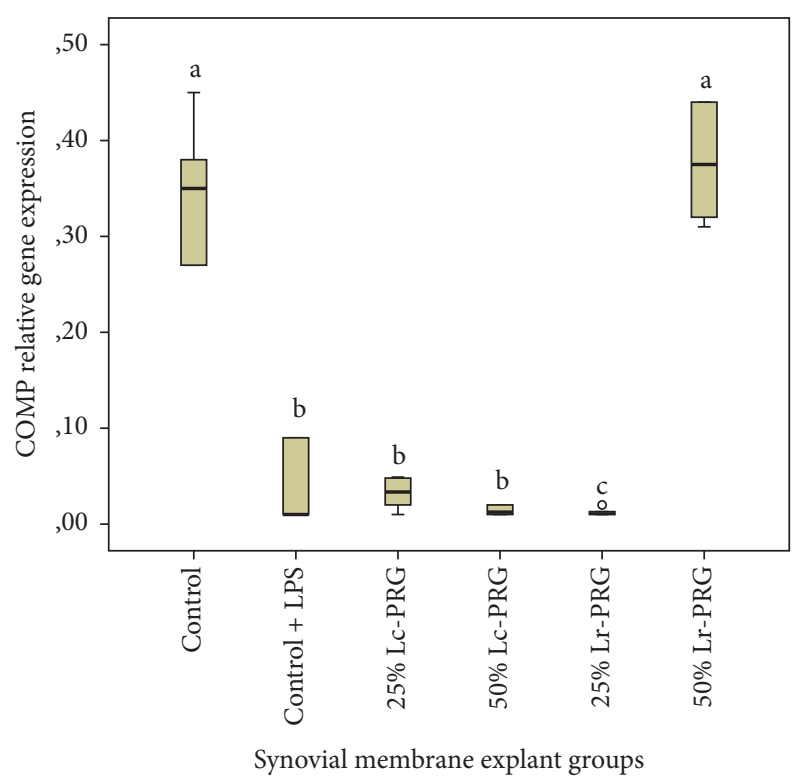

FIGURE 7: Box plot of median relative cartilage oligomeric matrix protein (COMP) gene expression. ${ }^{\mathrm{a}-\mathrm{c}}$ Lowercase letters denote significant differences $(p>0.05)$ between SME groups treated with Lc-PRG and Lr-PR supernatants at different concentrations (25 and $50 \%)$. Groups with the same lowercase letter are not significantly different. o denotes an outlier value, showing nonparametric data.

way of inflammation not dependent on NFKB gene expression. Notably, a similar molecular phenomenon has been described in synovial fibroblasts derived from rheumatoid arthritis patients in which MMP-13 upregulation was mediated by TGF- $\beta_{1}$ and laminin and not via NF $\kappa$ B upregulation [22]. However, it is also important to consider that perhaps some level of $\mathrm{NF} \kappa \mathrm{B}$ activity could play an important role in synovial homeostasis and its complete suppression could be unfavorable for joint health. In this sense, $\mathrm{NF} \kappa \mathrm{B}$ (at least in this in vitro system of synovitis) could be considered as a regulatory transcription factor and not as a proinflammatory gene. 
COL1A1 gene expression was significantly upregulated in SMEs of the control group challenged with LPS, but all PRG supernatants at both concentrations downregulated the expression of this gene in the SME groups challenged with LPS such that expression was very similar to the expression of this gene in the healthy SME control group. Similar findings also were found in a similar in vitro study using equine cartilage explants [12]. This finding is important because PRP preparations may reverse the formation of fibrocartilage in patients with OA or better yet in cases of traumatic arthritis and avoid synovial membrane fibrosis.

On the other hand, COL2A2 and COMP gene expressions were drastically downregulated in SMEs challenged only with LPS. The upregulation of these genes was completely reversed only by the $50 \%$ Lr-PRG supernatant. This last finding was similar for equine cartilage explants challenged with LPS and cultured with the same substances at a similar concentration [12]. However, the 25\% Lr-PRG supernatant led to better anabolic gene expression than the 50\% Lr-PRG supernatant in that study [12].

Synovial membrane is a source of mesenchymal stem cells, which always is available to release cells with chondrogenic potential. Consequently, these cells could promote joint cartilage repair or regeneration in cases of OA or other inflammatory arthropathies [23]. It is plausible that COL2A1 gene expression in SMEs is related to the chondrogenic differentiation of stem cells contained in these tissues. Interestingly, we find that LPS produced an intense downregulation of this gene, which was significantly marked for SMEs cultured with both Lc-PRG supernatants. Notably, $50 \%$ Lr-PRG supernatant exhibited a trend to counteract the COL2A1 downregulation (catabolic) effect of LPS in this in vitro system of synovitis, which could indicate that PRP (particularly Lr-PRP) preparations could be useful to induce chondrogenic differentiation of stem cells from synovial membrane in patients with OA $[24,25]$.

The results of this study are complementary to a previous similar in vitro study [4] in which we measured, in the culture medium of LPS-challenged SMEs at $48 \mathrm{~h}$ and $96 \mathrm{~h}$, the concentration of hyaluronan and some pro- (i.e., tumor necrosis factor alpha $(\mathrm{TNF}-\alpha))$ and anti-inflammatory (i.e., interleukin 4 (IL-4) and receptor antagonist of IL-1 (IL1ra)) cytokines. In that study, the $25 \%$ Lc-PRG supernatant induced the most robust concentration of IL-1ra, whereas $50 \%$ Lr-PRG induced the most sustained production of IL4 and hyaluronan [4]. These results, combined with those obtained in the present study, are useful to confirm that Lc-PRG supernatants at two concentrations can be classified as anti-inflammatory substances, possibly mediated by increased production of IL-1ra [4]; however, this antiinflammatory mechanism may not be able to induce synovial membrane anabolism. On the other hand, the 50\% LrPRG supernatant can be classified as an anti-inflammatory substance with intense anabolic effects, in which increased production of IL-4 could be involved [4]. It is known that this cytokine is able to induce cartilage ECM synthesis [26].

It is important to consider that although a healthy control group of SMEs was included, we decided to classify the therapeutic in vitro effect of the PRG supernatants as a function of their capacity to reverse the proinflammatory and catabolic effect of LPS on SMEs. Notably, none of the PRG supernatants (at both concentrations) evaluated in this study could produce a similar gene expression profile to what was observed in the healthy SMEs of the control group. At this point, the $50 \%$ Lr-PRG supernatant was able to induce the most similar gene expression profile when compared with the healthy SME group. These results have a very important biological value, because they demonstrate that LrPRG supernatants are able to counteract the proinflammatory and catabolic effects of LPS to some extent $[8,27]$.

Several correlations were observed in the present study. Notably, we observed that the expression of COMP and COL1A1 was negatively associated with the expression of $\mathrm{NF} \kappa \mathrm{B}$ and ADAMTS-4. In general, the 50\% Lr-PRG supernatant showed the most important anabolic effects, characterized by $\mathrm{NF} \kappa \mathrm{B}$ and ADAMTS- 4 downregulation and COMP upregulation. These findings are complementary to other PRP studies on joints [28, 29] and isolated cartilage [30] in which some researchers have concluded that Lr-PRP preparations are better to induce joint anabolism and to diminish inflammation than Lc-PRP preparations.

This study had several limitations, which should be addressed to avoid misinterpretation or exaggerated enthusiasm about the actual therapeutic potential of PRP in equine osteoarthritis or inflammatory (traumatic) arthritis. Autologous PRP preparations are frequently used for the treatment of equine patients with OA [31]. However, we used allogeneic PRP in the present study, which was obtained from blood of one donor mare. We processed the hemoderivatives (PRG supernatants) from the same animal in order to obtain substances with a standardized concentration of cells and proteins.

In general, the nature of this in vitro study was to evaluate the biological behavior of standard allogeneic hemoderivatives in the synovial tissues of genetically dissimilar horses to determine if these substances could affect the expression of selected genes implicated in OA. Moreover, this study could be useful to demonstrate that allogeneic PRP could be used for the treatment of OA horses. However, some of the beneficial effects observed in this system may have been influenced by immunological factors not associated with PRP.

It is known that one of the most important limitations for the dissemination of regenerative medicine products is the high costs related to the production of autologous products. Thus, there is constant interest in developing standardized and massive allogeneic biologic products free of pathogens and that do not induce immunologic rejections [32-34]. In line with this, it is necessary to evaluate the advantages and disadvantages of standardized allogeneic PRP preparations in treating musculoskeletal diseases in horses and humans [35].

\section{Conclusion}

This study demonstrates that both Lc- and Lr-PRP preparations at different concentrations can induce mixed antiinflammatory and anabolic responses in an in vitro system of equine synovitis. The $25 \%$ and $50 \%$ Lc-PRG supernatants presented slight to moderate anti-inflammatory effects. 
However, these substances did not reverse the catabolic effects of LPS on SMEs, since they were unable to induce the upregulation of anabolic genes. On the other hand, $25 \%$ and $50 \%$ Lr-PRG supernatants showed intense anti-inflammatory and anabolic effects, which were greater for the $50 \%$ Lr-PRG supernatant. Additional in vitro studies in a coculture system of cartilage and synovial membrane explants and in patients with OA should be performed to assess the in vivo effect of PRP preparations at different concentrations.

\section{Conflicts of Interest}

No potential conflicts of interest were reported by the authors.

\section{Acknowledgments}

This project was supported by the National Program of Biotechnology from COLCIENCIAS, Bogotá DC, Grant no. 397-2011, and the Vicerrectoría de Investigaciones y Posgrados of the Universidad de Caldas, Manizales, Colombia, Grant no. 0250911. The authors thank Wilson Gómez and Fabio Robayo for their assistance and acknowledge Policía Nacional de Colombia.

\section{References}

[1] H. N. Sadabad, M. Behzadifar, F. Arasteh, M. Behzadifar, and H. R. Dehghan, "Efficacy of Platelet-Rich Plasma versus Hyaluronic Acid for treatment of Knee Osteoarthritis: a systematic review and meta-analysis," Electronic Physician, vol. 8, no. 3, pp. 2115-2122, 2016.

[2] P. M. Brossi, J. J. Moreira, T. S. Machado, and R. Y. Baccarin, "Platelet-rich plasma in orthopedic therapy: a comparative systematic review of clinical and experimental data in equine and human musculoskeletal lesions," BMC Veterinary Research, vol. 11, no. 1, pp. 98-148, 2015.

[3] M. H. Mirza, P. Bommala, H. A. Richbourg, N. Rademacher, M. T. Kearney, and M. J. Lopez, "Gait Changes Vary among Horses with Naturally Occurring Osteoarthritis Following Intra-articular Administration of Autologous Platelet-Rich Plasma," Frontiers in Veterinary Science, vol. 3, 2016.

[4] D. L. Rios, C. López, M. E. Álvarez, I. J. Samudio, and J. U. Carmona, "Effects over time of two platelet gel supernatants on growth factor, cytokine and hyaluronan concentrations in normal synovial membrane explants challenged with lipopolysaccharide," BMC Musculoskeletal Disorders, 2015.

[5] P. Tyrnenopoulou, N. Diakakis, M. Karayannopoulou, I. Savvas, and G. Koliakos, "Evaluation of intra-articular injection of autologous platelet lysate (PL) in horses with osteoarthritis of the distal interphalangeal joint," Veterinary Quarterly, vol. 36, no. 2, pp. 56-62, 2016.

[6] S. Broeckx, M. Zimmerman, S. Crocetti et al., "Regenerative therapies for equine degenerative joint disease: a preliminary study," PLoS ONE, vol. 9, no. 1, Article ID e85917, 2014.

[7] E. Knop, L. E. Paula, and R. Fuller, "Platelet-rich plasma for osteoarthritis treatment," Revista Brasileira de Reumatologia (English Edition), vol. 56, no. 2, pp. 152-164, 2016.

[8] D. L. Rios, C. López, and J. U. Carmona, "Evaluation of the antiinflammatory effects of two platelet-rich gel supernatants in an in vitro system of cartilage inflammation," Cytokine, vol. 76, no. 2, pp. 505-513, 2015.
[9] D. M. D. Ehrenfest, I. Andia, M. A. Zumstein, C.-Q. Zhang, N. R. Pinto, and T. Bielecki, "Classification of platelet concentrates (Platelet-Rich Plasma-PRP, Platelet-Rich Fibrin-PRF) for topical and infiltrative use in orthopedic and sports medicine: current consensus, clinical implications and perspectives," Muscles, Ligaments and Tendons Journal, vol. 4, no. 1, pp. 3-9, 2014.

[10] D. M. D. Ehrenfest, T. Bielecki, R. Jimbo et al., "Do the fibrin architecture and leukocyte content influence the growth factor release of platelet concentrates? An evidence-based answer comparing a pure Platelet-Rich Plasma (P-PRP) gel and a leukocyte- and Platelet-Rich Fibrin (L-PRF)," Current Pharmaceutical Biotechnology, vol. 13, no. 7, pp. 1145-1152, 2012.

[11] E. Assirelli, G. Filardo, and E. Mariani, "Effect of two different preparations of platelet-rich plasma on synoviocytes," Knee Surgery, Sports Traumatology, Arthroscopy, 2014.

[12] J. U. Carmona, D. L. Ríos, C. López, M. E. Álvarez, J. E. Pérez, and M. E. Bohórquez, "In vitro effects of platelet-rich gel supernatants on histology and chondrocyte apoptosis scores, hyaluronan release and gene expression of equine cartilage explants challenged with lipopolysaccharide," BMC Veterinary Research, vol. 12, no. 1, article 135, 2016.

[13] H. J. Braun, H. J. Kim, C. R. Chu, and J. L. Dragoo, “The effect of platelet-rich plasma formulations and blood products on human synoviocytes: Implications for intra-articular injury and therapy," The American Journal of Sports Medicine, vol. 42, no. 5, pp. 1204-1210, 2014.

[14] G. Filardo, E. Kon, M. T. Pereira Ruiz et al., "Platelet-rich plasma intra-articular injections for cartilage degeneration and osteoarthritis: single-versus double-spinning approach," Knee Surgery, Sports Traumatology, Arthroscopy, vol. 20, no. 10, pp. 2082-2091, 2012.

[15] D. Arguelles, J. U. Carmona, J. Pastor et al., "Evaluation of single and double centrifugation tube methods for concentrating equine platelets," Research in Veterinary Science, vol. 81, no. 2, pp. 237-245, 2006.

[16] C. R. Byron, M. W. Orth, P. J. Venta, J. W. Lloyd, and J. P. Caron, "Influence of glucosamine on matrix metalloproteinase expression and activity in lipopolysaccharide-stimulated equine chondrocytes," American Journal of Veterinary Research, vol. 64, no. 6, pp. 666-671, 2003.

[17] M. N. Penha-Goncalves, D. E. Onions, and L. Nicolson, "Cloning and sequencing of equine transforming growth factorbeta 1 (TGF $\beta-1)$ cDNA," Mitochondrial DNA, vol. 7, no. 6, pp. 375-378, 1997.

[18] B. P. Donnelly, A. J. Nixon, J. L. Haupt, and L. A. Dahlgren, "Nucleotide structure of equine platelet-derived growth factor$\mathrm{A}$ and -B and expression in horses with induced acute tendinitis," The American Journal of Veterinary Research, vol. 67, no. 7, pp. 1218-1225, 2006.

[19] C. E. Giraldo, M. E. Álvarez, and J. U. Carmona, "Effects of sodium citrate and acid citrate dextrose solutions on cell counts and growth factor release from equine pure-platelet rich plasma and pure-platelet rich gel," BMC Veterinary Research, vol. 11, no. 1, article 60, 2015.

[20] C. E. Giraldo, C. López, M. E. Álvarez, I. J. Samudio, M. Prades, and J. U. Carmona, "Effects of the breed, sex and age on cellular content and growth factor release from equine pure-platelet rich plasma and pure-platelet rich gel," BMC Veterinary Research, vol. 9, article 29, 2013. 
[21] K. J. Livak and T. D. Schmittgen, "Analysis of relative gene expression data using real-time quantitative PCR and the $2^{-\Delta \Delta C T}$ method," Methods, vol. 25, no. 4, pp. 402-408, 2001.

[22] W. Aicher, T. Pap, and S. Gay, "Laminin-modulierte Entzündungsreaktionen in synovialen Fibroblasten von Rheumapatienten," Zeitschrift für Rheumatologie, vol. 67, no. 1, pp. 59-61, 2008.

[23] R. Sadatsuki, H. Kaneko, M. Kinoshita et al., "Perlecan is required for the chondrogenic differentiation of synovial mesenchymal cells through regulation of Sox9 gene expression," Journal of Orthopaedic Research, 2016.

[24] H. Tang, W. Chen, C. Chiang, L. Chen, Y. Chang, and C. Chen, "Differentiation effects of platelet-rich plasma concentrations on synovial fluid mesenchymal stem cells from pigs cultivated in alginate complex hydrogel," International Journal of Molecular Sciences, vol. 16, no. 8, pp. 18507-18521, 2015.

[25] L. C. Hermeto, R. DeRossi, R. J. Oliveira et al., "Effects of intraarticular injection of mesenchymal stem cells associated with platelet-rich plasma in a rabbit model of osteoarthritis," Genetics and Molecular Research, vol. 15, no. 3, Article ID gmr.15038569, 2016.

[26] S. J. Millward-Sadler, M. O. Wright, L. W. Davies, G. Nuki, and D. M. Salter, "Mechanotransduction via integrins and interleukin- 4 results in altered aggrecan and matrix metalloproteinase 3 gene expression in normal, but not osteoarthritic, human articular chondrocytes," Arthritis \& Rheumatism, vol. 43, no. 9, pp. 2091-2099, 2000.

[27] D. M. Bolt, A. Ishihara, S. E. Weisbrode, and A. L. Bertone, "Effects of triamcinolone acetonide, sodium hyaluronate, amikacin sulfate, and mepivacaine hydrochloride, alone and in combination, on morphology and matrix composition of lipopolysaccharide-challenged and unchallenged equine articular cartilage explants," American Journal of Veterinary Research, vol. 69, no. 7, pp. 861-867, 2008.

[28] Z. Xu, W. Yin, Y. Zhang et al., "Comparative evaluation of leukocyte- and platelet-rich plasma and pure platelet-rich plasma for cartilage regeneration," Scientific Reports, vol. 7, p. 43301, 2017.

[29] W.-J. Yin, H.-T. Xu, J.-G. Sheng et al., "Advantages of pure platelet-rich plasma compared with leukocyte- and platelet-rich plasma in treating rabbit knee osteoarthritis," Medical Science Monitor, vol. 22, pp. 1280-1290, 2016.

[30] E. A. Sundman, B. J. Cole, V. Karas et al., "The anti-inflammatory and matrix restorative mechanisms of platelet-rich plasma in osteoarthritis," American Journal of Sports Medicine, vol. 42, no. 1, pp. 35-41, 2014.

[31] J. U. Carmona, D. Argüelles, F. Climent, and M. Prades, "Autologous platele $\mathrm{t}$ concentrates as a treatment of horses with osteoarthritis: a preliminary pilot clinical study," Journal of Equine Veterinary Science, vol. 27, no. 4, pp. 167-170, 2007.

[32] C. Premer, A. Blum, M. A. Bellio et al., "Allogeneic mesenchymal stem cells restore endothelial function in heart failure by stimulating endothelial progenitor cells," EBioMedicine, vol. 2, no. 5, pp. 467-475, 2015.

[33] J. M. Hare, J. E. Fishman, G. Gerstenblith et al., "Comparison of allogeneic vs autologous bone marrow-derived mesenchymal stem cells delivered by transendocardial injection in patients with ischemic cardiomyopathy: the POSEIDON randomized trial," JAMA, vol. 308, no. 22, pp. 2369-2379, 2012.

[34] P. F. van der Meer, J. Seghatchian, and D. C. Marks, "Quality standards, safety and efficacy of blood-derived serum eye drops: a review," Transfusion and Apheresis Science, vol. 54, no. 1, pp. 164-167, 2016.

[35] T. Kawase, "Platelet-rich plasma and its derivatives as promising bioactive materials for regenerative medicine: basic principles and concepts underlying recent advances," Odontology, vol. 103, no. 2, pp. 126-135, 2015. 

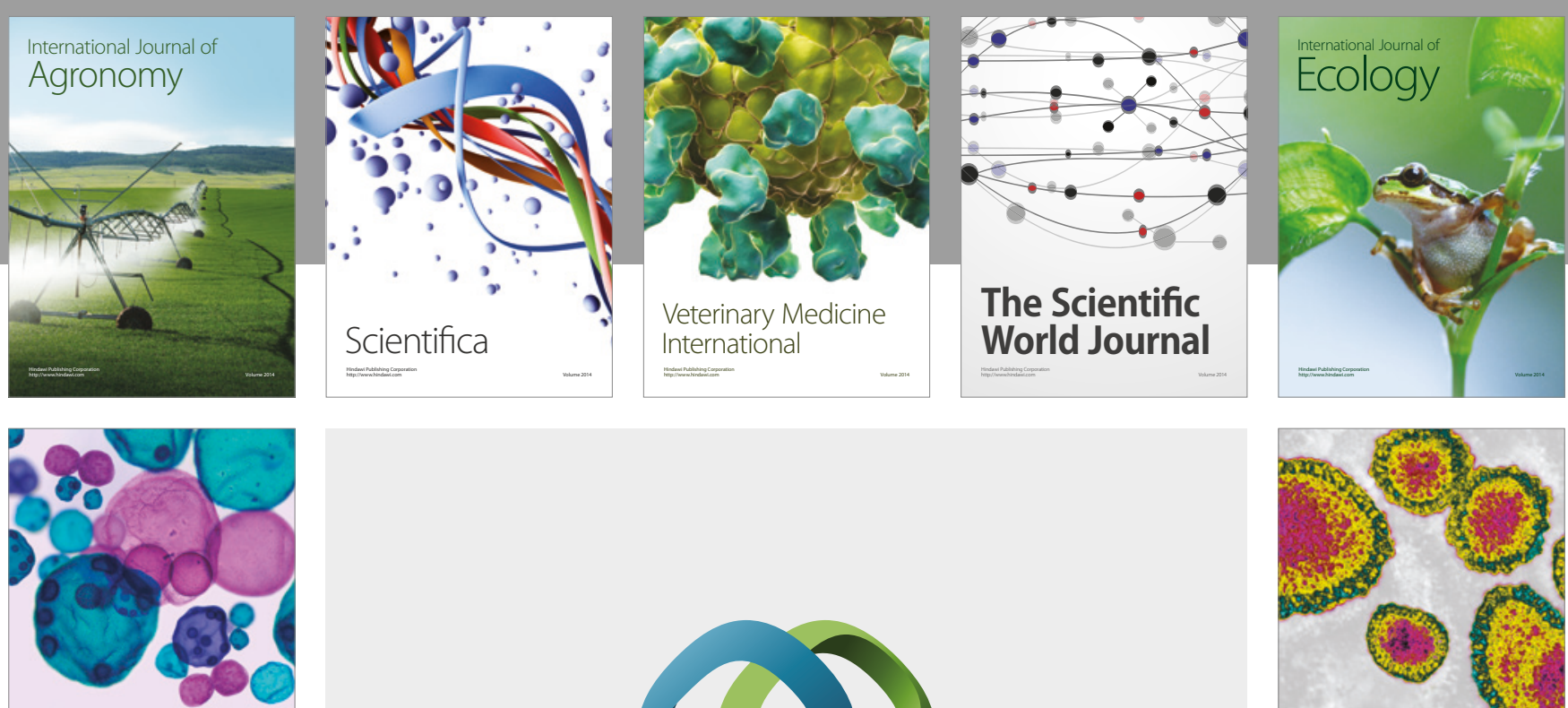

\section{International Journal of} Microbiology
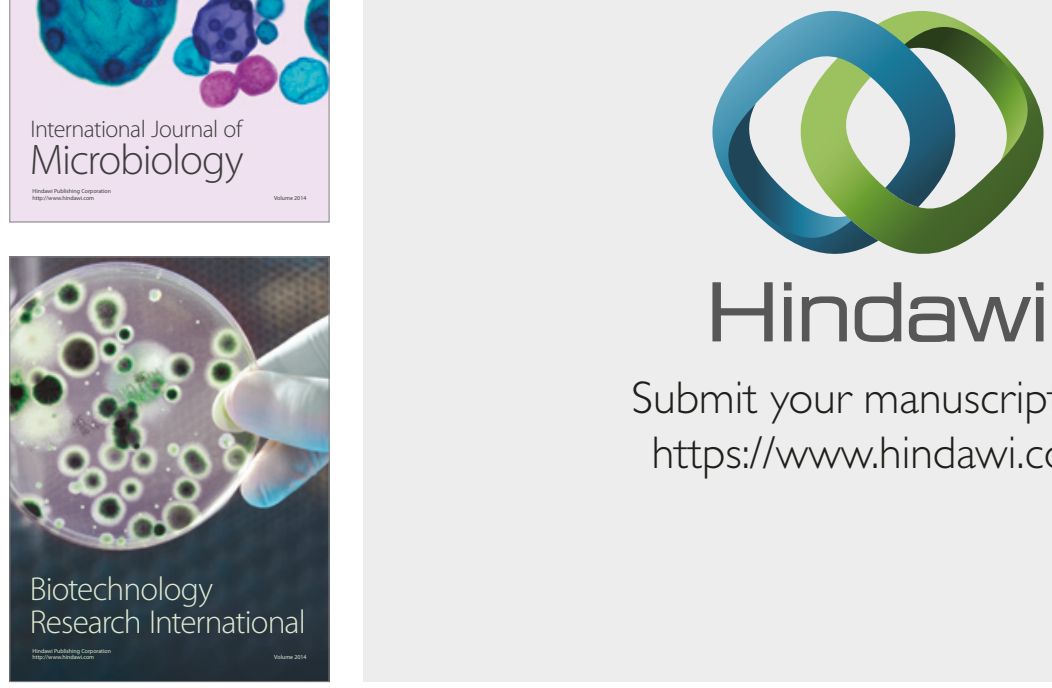

Submit your manuscripts at

https://www.hindawi.com
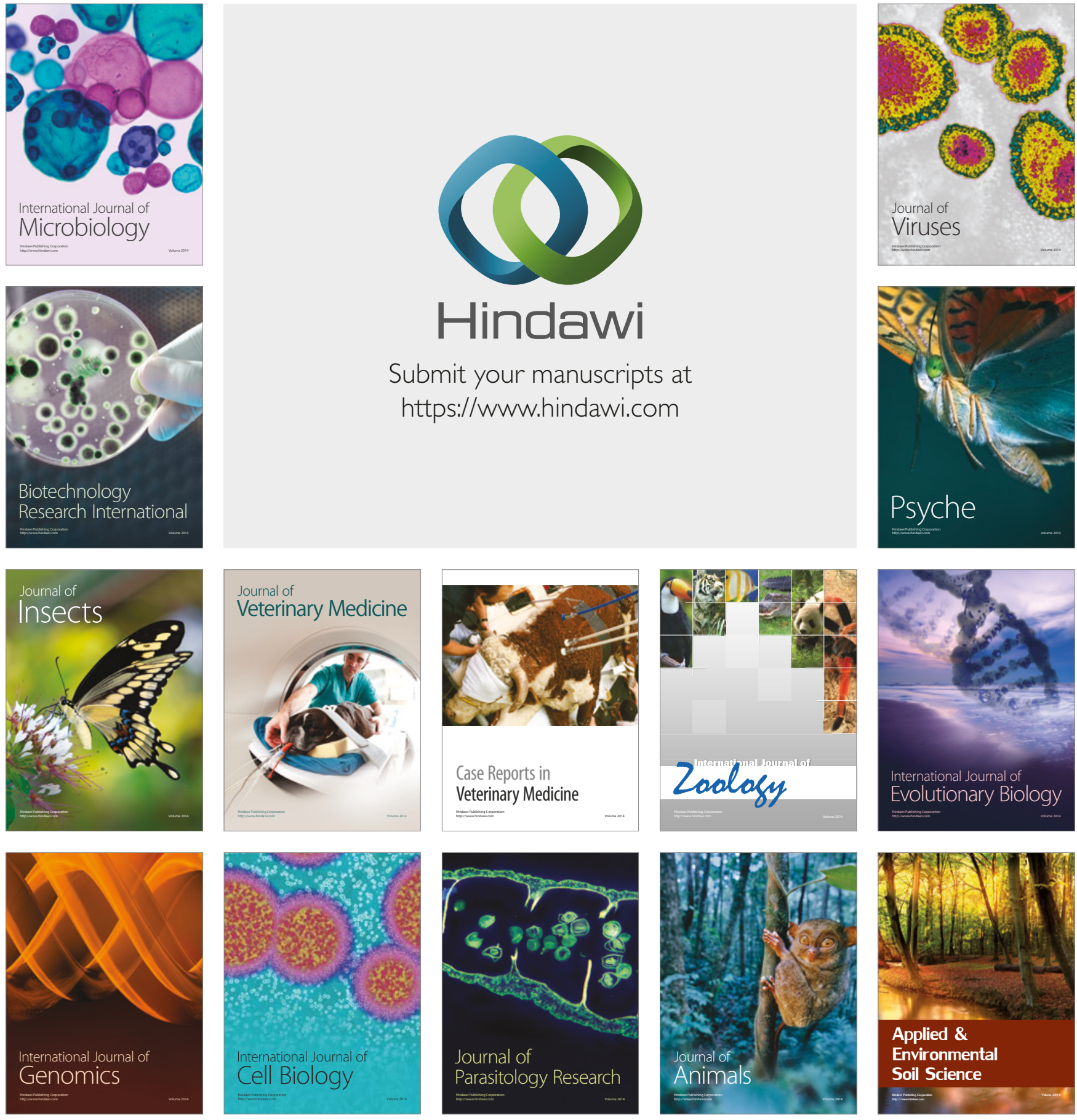